\title{
Value-based Engineering: Prinzipien und Motivation für bessere IT-Systeme
}

\author{
Sarah Spiekermann ${ }^{1}$ \\ Angenommen: 10. Juni 2021 / Online publiziert: 29. Juli 2021
}

@ Der/die Autor(en) 2021

\section{Zusammenfassung}

Digitale Ethik wird derzeit weltweit als eine Notwendigkeit diskutiert, um verlässlichere IT-Systeme zu schaffen. Diese Diskussion, geschürt durch die Angst vor einer unkontrollierbaren generellen Künstlichen Intelligenz (KI) und angeheizt von ethischen Dilemmata bestehender Systeme, hat viele Institutionen und Wissenschaftler dazu bewegt, Werteprinzipien einzufordern, an denen sich die Entwicklung komplexer Systeme zukünftig orientieren sollte. Diese inkludieren i.d.R. die Forderung nach Privatsphäre, Sicherheit, Transparenz, Fairness etc. In diesem Artikel wird dargestellt, warum das Abarbeiten von Wertelisten für eine gute bzw. ethisch legitimierte Systementwicklung unzureichend ist. Es wird gezeigt, wie stattdessen ein wirklich ethisches „Value-based Engineering“ aussehen müsste, damit technische Produktinnovationen insgesamt auf bessere (ethischere) Füße gestellt werden. Value-based Engineering (VbE) ist eine prozessgetriebene, ganzheitliche Herangehensweise an die Systementwicklung, die zunächst aus den Ideen des Value Sensitive Designs und dem Ethical Computing schöpfte. Ab 2016 wurde VbE im IEEE 7000 Standard weiter konkretisiert. 10 Prinzipien für ethisch angeleitete Systeminnovation fassen wesentliche Handlungsanforderungen für Unternehmen zusammen.

Der offizielle Link zum IEEE 7000 lautet: https://standards.ieee. org/project/7000.html. Die IEEE Working Group (WG) wurde von 2016 bis 2021 von der Autorin dieses Artikels als Vice-Chair mit geleitet und umfasste offiziell mehr als 30 Experten mit unterschiedlichem fachlichen Hintergrund, hauptsächlich Software- und Systemingenieure, aber auch einige Volontäre mit Fachwissen in Philosophie. Die Grundlage für die Diskussionen dieser Experten war ein Standardentwurf, der von der Autorin dieses Artikels verfasst wurde und der eine frühe Version von $\mathrm{VbE}$ enthielt, die im Lehrbuch „Ethical IT Innovation A Value-based System Design Approach“ nachgelesen werden kann [22]. Dieser Artikel profitiert von den Expertengesprächen in der Standardisierungsgruppe. Dieser Artikel gibt dennoch ausschließlich die Meinung der Autorin wieder und repräsentiert nicht notwendigerweise eine Position der IEEE P7000 Working Group, des IEEE oder der IEEE Standards Association. Weitere Informationen $\mathrm{zu} \mathrm{VbE}$, wissenschaftliche Artikel, Tools und Lehrmaterialien können über https://www.wu.ac.at/value-basedengineering bezogen werden.

\section{Sarah Spiekermann}

mis-sek@wu.ac.at

1 Wirtschaftsuniversität Wien, Wien, Österreich

\section{Einleitung}

In den Jahren seit ca. 2015 ist das Bewusstsein für eine ethischere Gestaltung von Technologie kontinuierlich angestiegen. In Deutschland war es möglicherweise der Volkswagen-Skandal (2015), der wesentlich zum Hinterfragen ethischer Praktiken in klassischen „Engineering“-Abteilungen der Industrie in einer breiten Öffentlichkeit beigetragen hat. Kurz darauf, im Jahr 2016, stimmten die Briten für den Brexit und Donald Trump wurde in den USA zum Präsidenten gewählt. Analysen aus dem Bereich des investigativen Journalismus zeigten seitdem, dass beide politischen Entscheidungen der Bürger über soziale Netzwerke massiv manipuliert worden sind; ein Manipulationsvorgang, der bei näherer Betrachtung nur möglich war, weil die technische Architektur der sozialen Plattformen (insbesondere Facebook in diesem Fall), die Schnittstellen (zu Englisch: Application Programming Interfaces oder APIs), die Authentifizierungsprozesse, die systematische Sammlung, Aggregation und Verfügbarmachung persönlicher Daten und Profile dies zuließen. Gleichzeitig mehren sich die Meldungen von fehlgeleiteten KI-Systemen, die Richter (vor allem in USA) zu falschen Vorurteilen (,biases“) verleiten [20] oder Universitätsbewerbern falsche Qualifikationen zuschreiben [10]. Dies ist möglich, da selbstlernende Systeme etwa nicht transparent genug arbeiten, schlichtweg 
für andere Zwecke trainiert wurden oder das System keine geeignete Datenbasis bzw. Datenqualität hat. Immer mehr wird der IT-Industrie und ihren Kunden bewusst, dass eine vorausschauendere, verantwortlichere und ethisch systematischere Planung von IT-Systemen erforderlich ist, damit Probleme nicht in solchem Ausmaß entstehen. Werte wie Privacy (Datenschutz), Sicherheit, Transparenz, Verantwortung, Kontrolle usw. sind als Themen für das Engineering vor diesem Hintergrund höher im Kurs als je zuvor. So hoch sogar, dass die Gartner-Analysten begonnen haben, Technologietrends hervorzuheben, die Wertprädikate besitzen;

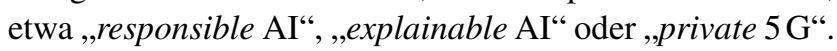

\section{Ethik in der Systementwicklung heute: Passivität und Wertelisten}

Doch wie kommt dieser Hype um ,ethischere“ Technik in der Praxis an und wie wird er von den regulären Unternehmensprozessen aufgefangen? Eine übliche Herangehensweise an das Thema Ethik in der Alltagspraxis der Systementwicklung ist leider immer noch eher passiv. Abgesehen von einem gelegentlichen Austausch mit der Corporate-Social-Responsibility(CSR)-Abteilung, beschäftigt man sich in technischen Unternehmenseinheiten oft erst dann mit der Ethik, wenn es ein nicht zu ignorierendes Problem gibt; etwa wenn das Corporate Audit Sicherheitslücken identifiziert oder wenn die Datenschutzgrundverordnung Schritte in Richtung mehr User Privacy fordert. Sicherlich wird Boeing sich jetzt mehr denn je mit der Frage beschäftigen, wie viel Kontrolle Piloten wirklich brauchen und wie es mit der Transparenz der technischen Abläufe im Cockpit aussieht. Unternehmen werden häufig erst dann aktiv, wenn Feuer gelöscht werden muss, wenn ein neues Gesetz erfüllt werden muss oder wenn Werte bereits so massiv verletzt wurden, dass Strafen, schlechte Presse und Kundenbeschwerden ins Haus stehen.

Anders sieht es in den letzten Jahren auf strategischer Unternehmensebene aus oder in politischen Kreisen. Hier überschlagen sich die Angebote an vielversprechenden Listen von Werteprinzipien, die zukünftig vom Unternehmen gelebt und von der Technik umgesetzt werden sollen. Beispiele sind IBM's Prinzipien der ,Everyday ethics for $\mathrm{AI}^{\text {“ }}$ oder Microsofts „Responsible AI“-Werteliste. Jüngst präsentierte das Autorenteam rund um Ann Jobin et al. [13] im Journal Nature Machine Intelligence eine Analyse von 84 institutionell verabschiedeten Wertelisten, die insbesondere komplexere Systeme betreffen, die unter der Bezeichnung „,künstliche Intelligenz“ geführt werden [13]. In diesen Listen kommen Wertversprechen sowohl von Unternehmen als auch von politischen Institutionen (z. B. der OECD) und globalen Verbänden (z. B. IEEE) zur Sprache. Die Hoffnung steht im Raum, dass durch solche öffentlichen Feststellungen, Guidelines und auch Commitments zu bestimmten Werten ein generell gesteigertes Bewusstsein für die Ethik in der Systementwicklung entsteht. Sollten die 5 häufigsten Werte, die in der Analyse von Jobin et al. [13] als Gemeinsamkeiten identifiziert wurden - Transparenz, Fairness, Unbedenklichkeit, Verantwortlichkeit, Datenschutz - als Hygienefaktoren guter Systementwicklung in jedem System-Engineering-Lehrbuch und Start-up erklärt, gelehrt, verstanden und umgesetzt werden, dann wäre das sicherlich ein großer Fortschritt in Richtung einer menschenfreundlicheren und sozialeren Digitalisierung.

Transparenz - steht für die angemessene, zugängliche, verständliche, bedeutungsrelevante und ehrliche Dokumentation und Kommunikation, wie ein System funktioniert und wie es Entscheidungen trifft (S. 59 in [22]).

Fairness - steht für die Wahrung von Gleichbehandlung der Nutzer und Abwesenheit systematischer Voreingenommenheit der KI (auf Englisch: ,absence of bias“) sowie gleichberechtigte Zugänglichkeit des Systems (auf Englisch: ,accessibility“).

Unbedenklichkeit - steht vor allem für die verschiedenen Dimensionen der Systemsicherheit.

Verantwortlichkeit - steht für Formen der Rechenschaftslegung, Haftung und Integrität der Handlungen der Systemverantwortlichen.

Datenschutz - steht für die in der europäischen Datenschutzgrundverordnung verankerten Prinzipien, wie etwa das Recht auf Vergessenheit, die Datenportabilität, die informierte Zustimmung etc.

Aber ist der Ethik damit Genüge getan? Wie weit bringt uns die Compliance mit solchen Listen von Wertprinzipien?

In diesem Artikel werde ich zunächst darstellen, warum Wertelisten bei aller guten Absicht der Autoren auch zur Verkennung der ethisch eigentlich relevanten Herausforderungen für einen Systemkontext führen können und stelle stattdessen die 10 Handlungsprinzipien vor, derer es bedarf um ,gute“ IT Systeme zu bauen (Zusammenfassung Abb. 2).

\section{Wertelisten verführen zur Verkennung des ethisch Relevanten}

In meinem Buch Digitale Ethik - Ein Wertesystem für das 21. Jahrhundert beschreibe ich ausführlich das Beispiel, wie das Wertedesign von Sprachassistenten auf subtile Weise völlig anders sein kann, je nachdem in welcher Kultur diese entwickelt werden. Auf die Aussage des Nutzers eines US-amerikanischen Alexa-Systems "Ich bin traurig“, antwortete Alexa in 2017 ,Ich wünschte, ich hätte Arme, um Dich zu knuddeln.“ Das russische System Yandex hingegen antwortete „Keiner hat gesagt, dass das Leben eine Spaßveranstaltung ist“ (S. 176 [23]). 
Beim Lesen dieser beiden völlig unterschiedlichen Antworten stellt sich die Frage: Haben die Entwickler der Dialogsysteme über die ethischen Implikationen ihrer beiden Systeme eigentlich bewusst nachgedacht? Haben sie sich damit beschäftigt, dass die Antwort bestimmte Werte zum Ausdruck bringt und dass sie - je nach Markterfolg und Diffusion des Systems - daher die Nutzer (z. B. Kinder) in ihrer Haltung zum Leben oder in ihrer Entwicklung durchaus beeinflussen könnten? Überhaupt: Welche Antwort wäre eigentlich die richtigere, die amerikanische oder die russische? Das Nachdenken und Debattieren über diese letzte Frage geht ins Herz einer ethischen Auseinandersetzung mit dem technischen Ding: „Wie soll ich handeln?“ [15]. Ich, der Ingenieur des Dialogsystems. Wie gebe ich dem Sprachassistenten den ,richtigen“, „guten“ oder „weisen“ Dialog mit? Die Antwort auf diese Frage ist eine Funktion der Werte, die man erfahrbar machen möchte. Bei dem gegebenen Beispiel steht zur Diskussion, ob der Sprachassistent eher Werte wie die Tugend der mentalen Abhärtung, die Persönlichkeitsrobustheit und Disziplin fördern sollte oder aber auf das Wohlfühlen, die Vermittlung von Nähe und das Mitgefühl. Die Möglichkeit, das zu entschieden und so über ein Dialogsystem wertethisch auf die Gesellschaft einzuwirken, ist eine ungeheuer gestalterische und bereichernde Herausforderung. Eine Herausforderung, die von Innovationsteams in Unternehmen mit großem Interesse und Verantwortungsgefühl gelebt werden könnte, wäre dafür nur genug Zeit und Raum gegeben. „Value-based Engineering“ soll diese Zeit und diesen Raum schaffen. Es stellt Prozesse zur Verfügung, die Systementwicklungsteams und Führungskräften dabei helfen sollen, ganz offiziell in genau diese Art von Wertreflektion einzusteigen. Es geht darum, die feingranulare Wertstruktur zukünftiger Systeme zu antizipieren und ganz bewusst im Interesse von Stakeholdern zu durchdenken. Anschließend werden die identifizierten Werte priorisiert und mithilfe von sogenannten „Ethical Value Requirements“ (EVRs) in Systemanforderungen übersetzt [12].

Wie das Beispiel des Sprachassistenten zeigt, überschneiden sich jedoch die für die Technologie spezifisch relevanten Werte nicht unbedingt mit den oben beschriebenen und global ausgerufenen Wertelisten. Diese Nichtüberlappung von dem, was ethisch wesentlich ist für ein „System of Interest“ (SOI) im Kontext - wie hier das Trösten des Users - und dem, was global als wesentlich gelistet ist, ist signifikant. Obgleich die öffentliche Benennung und das Bekenntnis zu bestimmten technischen Hygienewerten ungeheuer wichtig sind, weil sie ein Bewusstsein für diese ebenso fördern wie eine technische Auseinandersetzung mit deren Erfüllung, so sind sie doch bei Weitem nicht ausreichend. Der breite Fächer von menschlich- und sozial relevanten Werten, die unsere Interaktion mit IT-Systemen durchweben, wird durch die Wertelisten leicht verkannt.
In dem Verb des „Verkennens“ drückt sich eine Gefahr aus, die bei der ethischen Systementwicklung unbedingt vermieden werden sollte: Wertelisten sind nicht nur zu kurz. Sie können auch die Auseinandersetzung mit den wahren Herausforderungen eines SOI ,primen“. So primen, dass die eigentlich relevanten wertethischen Aspekte eines Systems vernachlässigt werden. So identifizierte etwa eine Gruppe von Innovationsmanagementstudenten der Wirtschaftsuniversität Wien beim Durchdenken eines „Location-Tracking"-Systems für die Verkaufsfläche eines Modehauses für Senioren den Wert der Kundenprivatsphäre als die wichtigste ethische Herausforderung des Systems. Orientierung bot ihnen bei dieser Analyse u.a. eine Werteliste, in der Privatsphäre enthalten war. Eine andere Studentengruppe, die eine solche Liste nicht nutzte, sondern stattdessen das Modehaus besuchte und sich mit den älteren Herrschaften in dem weitläufigen und vollgestopftem Outlet unterhielt, erfuhr hingegen, dass der wesentlichste Kernwert, den ein Location Tracking überhaupt bieten könnte, im Wert der „Hilfe“ bestünde. Sie erfuhren, dass der Wert der Privatsphäre in dem Moment für die Senioren sekundär wird, in dem sie (die Kunden) für das Teilen ihrer Standortdaten einen echten Kernwert in Form von Hilfe zurückerhalten. Hilfe etwa dadurch, dass das System Wertqualitäten wie den schnellen Zugang zu Verkäufern bietet (wo sind diese im Verhältnis zu meinem Standort im Laden?), eine verbesserte Orientierung im Laden erlaubt (wo ist mein Standort im Verhältnis zur Ware, die ich suche?) oder Zeitersparnis bringt (wie komme ich am schnellsten zur gesuchten Ware?). Value-based Engineering, so wie es im IEEE 7000 beschrieben ist, fordert Innovationsteams auf, in Interaktion mit Stakeholdern (hier den Senioren) zunächst frei von Wertelisten über mögliche Wertedynamiken (Kernwerte und Wertqualitäten) im Kontext eines SOI nachzudenken. Diese Auseinandersetzung mit der unmittelbar erlebten oder imaginierten Einsatzwirklichkeit eines SOI erlaubt es, zu eruieren, zu fragen und zu erkennen, was für zukünftige direkte und indirekte Stakeholder von Bedeutung bzw. von Wert sein könnte. Und genau auf diese Wertigkeiten soll ein SOI dann in der Entwicklung abgestimmt werden.

Diese Herangehensweise einer unvoreingenommenen Auseinandersetzung mit der gegebenen Wertevielfalt eines SOI-Kontexts deckt sich mit den Empfehlungen vieler Vordenker, wie etwa Ikujiro Nonaka, der in seinen Arbeiten der Antizipation von Werten eine große Bedeutung zuspricht, um Innovationsanstrengungen erfolgreich zu gestalten [19]. Sie deckt sich auch mit den Arbeiten von Batya Friedman, die mit ihrem „Value-Sensitive-Design“-Ansatz 2 Jahrzehnte an Erfahrungen gesammelt hat, kontextrelevante Werte zu erkennen und in technischen Systemen zu berücksichtigen [7]. Friedman nutzt das Wort der „Sensitivität“ und es scheint, als signalisiere sie damit eine Haltung, die es uns 
erlaubt, mit den zu erwartenden Wertfolgen eines Systems verantwortlich umzugehen.

Dennoch mögen an dieser Stelle 2 kritische Fragen gestellt werden. Erstens: Was hat ein Wert wie die ,Hilfe ' in diesem Location-Tracking-Beispiel mit Ethik zu tun? Zweitens: De-priorisiert die Werterkennungsphase des VbE hier nicht einen rechtlich und moralisch anerkannten Wert wie die Privatsphäre hinter einem anwendungsnahen Wert wie der Kundenhilfe? Die folgenden Abschnitte werden diese Fragen beantworten.

\section{Ethische Grundlagen des Value-based Engineering}

Die materiale Wertethik von Max Scheler, auf der das VbE beruht, betrachtet alles was uns umgibt, andere Menschen, die Natur, Technik, Beziehungen oder Aktivitäten als potenziellen Wertträger [21]. Dinge wie ein Sprachassistent oder eine Location-Tracking-Anwendung können zu Trägern von Werten werden, wenn sie technische Konfigurationen als Potenzial bergen, die einen Wert ermöglichen. Die bewusste wertethische Dialogkonfiguration des Sprachassistentendialogs etwa ist die Voraussetzung dafür, dass sich für einen kindlichen Nutzer entweder der Wert der Nähe erschließt oder der Wert der Abhärtung. Für den Senior im Modehaus kann sich der Wert der Hilfe erschließen, wenn er auf die Location-App im Smartphone blickt und dort einen großen grünen Knopf sieht, der ihm erlaubt, Personal herbeizurufen. Die technische Konfiguration ist also eine Disposition oder eine Voraussetzung, die vom Systementwickler geschaffen werden kann und die als Potenzial zur Verfügung steht, damit Wert entstehen kann. Das Resultat für das Ökosystem an Interessensgruppen (englisch: „,stakeholder") - für die Kunden und Verkäufer in diesem Fall ist positiv. Es kommt zu einer Bereicherung auf beiden Seiten: der Hilfesuchenden und der Helfenden. Genau das aber, die Erschaffung bzw. Förderung eines positiv gestimmten Ökosystems für involvierte direkte und indirekte Stakeholder, ist das Anliegen des $\mathrm{VbE}$ in Anlehnung an die materiale Wertethik. Ein bewusstes und vorausschauendes Nachdenken über „Wertträger“, „Wertdispositionen“, „Kernwerte“ und „Wertqualitäten“ erlaubt es, ein Systemdesign von Anfang an wertstrategisch und damit ethisch zu denken. Ethik heißt hier im Anschluss an die materiale Wertethik also, die Umgebung als wertgeladen zu denken, zu sehen und zu gestalten.

\section{Nutzung der Moralphilosophie im Erkennen von Werten}

Wie stellt man sicher, dass eine Wertestrategie dabei auch wirklich ethisch ist? Vor allem dann, wenn man aus den besagten Gründen zunächst nicht auf vorgegebene, institutionell abgesegnete Wertelisten zurückgreift? Im VbE und im IEEE 7000 Standard wird erwartet, 3 anerkannte Moraltheorien im Kontext anzuwenden. In Bezug auf das jeweilige SOI und mit einem ersten Concept of Operation vor Augen werden 3 Fragen gestellt, die dem Utilitarismus [16], der Tugendethik [2] und der Pflichtethik [14] entlehnt sind:

1. Utilitarismus: Welche menschlichen, sozialen, ökonomischen oder sonstigen Werte sind im Positiven wie im Negativen durch das SOI tangiert?

2. Tugendethik: Wie wirkt sich die Technik langfristig auf den Charakter der betroffenen Stakeholder aus?

3. Pflichtethik: Welche persönlichen Maxime oder Wertprioritäten sieht das Projektteam durch den Service betroffen, die die Mitglieder des Projektteams für so wichtig halten, dass sie sie in der Gesellschaft bewahren möchten?

Durchdacht werden diese 3 Fragen nicht nur vom unmittelbar verantwortlichen Projektteam, welches das SOI bauen soll, sondern von einer breiten Gruppe von Stakeholderrepräsentanten, die in die Entwicklung der Wertstrategie eingebunden werden und deren Rolle es ist, eine kritische und ganzheitliche Sicht darauf zu nehmen. Im Stakeholderdiskurs werden - geleitet durch diese 3 Fragen - Ideen dazu gesammelt, wie sich das SOI auswirken könnte; und zwar sowohl im Guten wie im Schlechten. Der Diskurs zwischen den Stakeholdern und deren Auswahl sollte dabei Anforderungen gerecht werden, wie sie etwa von Jürgen Habermas in seiner Diskursethik angedacht [9] und in der IS-Forschung rezipiert worden sind [17]. Sollte in einer Kultur, in der das SOI zum Einsatz kommen soll, eine spezielle ethische Ausrichtung verankert sein, die über diese 3 ethischen Fragenschwerpunkte hinausgehen, so regt der IEEE 7000 Standard dazu an, für die jeweilige Kultur eine weitere relevante Frage hinzuzufügen.

Das Resultat einer solchen Reflektion der Werte ist in der Regel, dass selbst für einfache SOIs ein relativ breites Wertspektrum identifiziert wird, das potenziell von Bedeutung sein könnte. Über 3 Case Studies hinweg konnten wir empirisch beobachten, dass Stakeholder durchschnittlich 16-19 Werte als relevant identifizieren, was sich bei größeren Stakeholdergruppen zu einer beträchtlichen Anzahl von Werten summieren kann [3], selbst wenn sich einige Werte inhaltlich überschneiden. Für einen der 3 Cases, ein Wiener Telemedizin-Start-up, wurden von Innovationsmanagementstudenten insgesamt 54 einzigartige Wertverletzungen identifiziert, die durch die Plattform entstehen könnten, ebenso wie 63 positive Werte, die man mit einer Telemedizinplattform fördern könnte. Diese große Zahl an negativ wie positiv relevanten Werten macht bewusst, wie ethisch feingranular und sensibel eine Technologie eigent- 


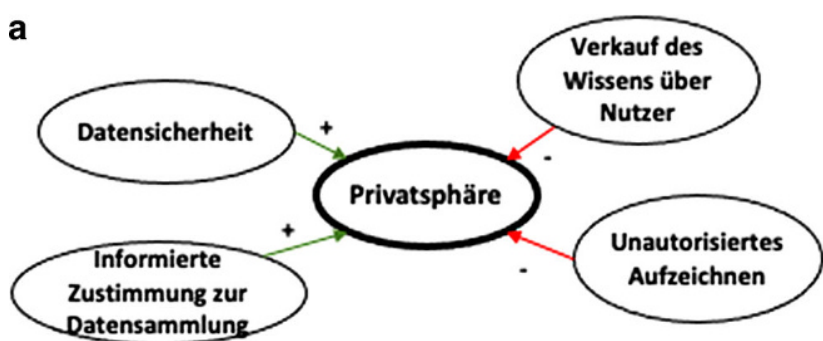

Abb. 1 a Kernwertcluster „Privatsphäre“, b Kernwertcluster „Trost“

lich wirken kann, wenn man sie mithilfe einer größeren kritischen Stakeholdergruppe durchdenkt. Das Freilegen der Wertpotenziale schärft jedoch nicht nur die ethische Sensibilität. Für Investoren wird auch klar, wie risikoreich eine neue Technologieinvestition wirklich ist. Und für das Produktmanagement erschließen sich durch die vielen positiven Wertpotenziale Hinweise, die „Value Proposition“ ihrer Geschäftsidee zu verfeinern.

Um die Komplexität des Wertspektrums zu bewältigen, werden alle Wertbeobachtungen strukturiert und als „Kernwerte“ mit „Wertqualitäten“ geclustert (siehe Abb. 1a,b). Kernwerte sind solche Werte, die immer wieder von den versammelten Stakeholdern beschrieben oder sogar direkt benannt werden und die daher ein besonderes Gewicht haben. Sie sollten als Kernwerte des zukünftigen Systems positiv formuliert sein und intrinsischer Natur sein. Das heißt, dass sie um ihrer selbst willen begehrt werden und dass selbst kulturübergreifend selten bezweifelt wird, wozu sie gut sind (z. B.: Freundschaft, Wissen, Sicherheit etc.) Davon abgegrenzt sind die für die Kernwerte instrumentalen Wertqualitäten, die sich aus den Umschreibungen der Stakeholder ebenso ergeben. So würde ein Stakeholder wenn es ihm um die Privatsphäre seines Sprachassistenten geht vielleicht sagen, dass er nicht möchte, dass das Wissen des Sprachassistenten über den Nutzer weiterverkauft werden darf und dass es zu keinem unautorisierten Aufzeichnen kommen sollte, dass die Datensicherheit gewährleistet sein muss und dass überhaupt für alle Datennutzung des Sprachassistenten eine Nutzerzustimmung vorliegen muss. Solche Aussagen der Stakeholder drücken die "Qualitäten“ des Kernwerts Privacy aus, die im SOI verankert werden sollten. $\mathrm{Zu}$ beachten ist, dass Wertqualitäten selbst auch wiederum Werte sein können (aber nicht müssen). Im IEEE 7000 Standard hat man sich anstelle von „Wertqualität“ auf die Bezeichnung „Value Demonstrator“ geeinigt. Die Arbeitsgruppe wollte damit ausdrücken, dass sich ein Kernwert in seinen Wertqualitäten ,zeigt“ (,demonstrates itself in").

Schließlich sei hinzugefügt, dass die im Stakeholderprozess gesammelten Wertqualitäten meistens nicht vollständig sind. Stakeholder haben einen intuitiven Zugang zu Werten. Sie erkennen diese in Schelers Worten durch ihre „Wertge-

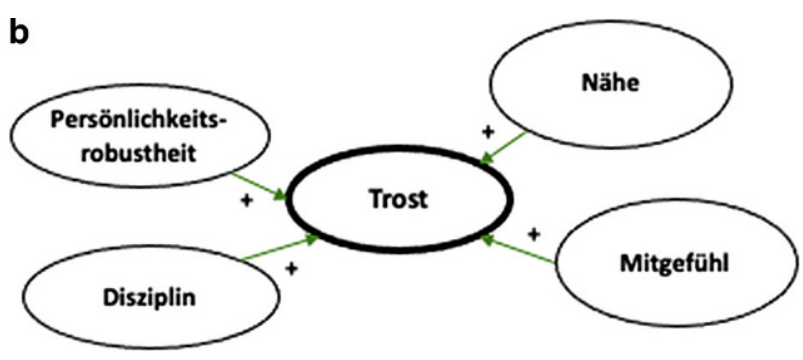

fühle“ (S. 265 ff in Scheler [21]). Zum Beispiel können Stakeholder tatsächlich Angst vor dem Verlust ihrer Privatsphäre im Umgang mit Sprachassistenten empfinden [8]. Aber Gefühle reichen in der Regel nicht aus, um einen Kernwert konzeptionell vollständig zu erfassen. Abb. 1a zeigt dies deutlich. Wer die in der Datenschutzgrundverordnung berücksichtigten Prinzipien zur Gewährleistung von Privatsphäre kennt, weiß, dass es deutlich mehr gesetzlich verankerte Qualitäten gibt [6] als die, welche Stakeholder hier diskutiert haben (Abb. 1a). Aus diesem Grund sieht das VbE ebenso wie das Value Sensitive Design vor, dass eine sogenannte ,conceptual analysis“ erfolgen muss [7], die im VbE so umgesetzt wird, dass priorisierte Kernwertcluster um weitere, etwa gesetzlich anerkannte Wertqualitäten ergänzt werden.

\section{Nutzung der Moralphilosophie zur Priorisierung von Werten}

Im Fall des Telemedizin-Start-ups konnten 13 originäre Kernwertcluster identifiziert werden. Welche sollen berücksichtigt und in einer konzeptionellen Analyse vertieft werden? Das VbE und IEEE 7000 sehen nicht vor, dass Kernwerte gegeneinander ausgespielt werden; dass sie also als eine Art „Trade-off“ behandelt werden. Stattdessen werden die Cluster Führungskräften und Stakeholderrepräsentanten vorlegt, damit diese sie in ihrer Bedeutung für das SOI reihen. Bei dieser Reihung werden folgende Kriterien berücksichtigt [12], die als gleichbedeutend angesehen werden:

1. Die Stakeholder streben an, dass durch die Reihung der Werte das SOI gut für die Gesellschaft sein wird und Wertverletzungen vermieden werden.

2. Die Organisation berücksichtigt bei der Priorisierung der Werte, dass die vom SOI betroffenen Menschen nicht nur als Mittel zum Zweck behandelt werden.

3. Die Führungskräfte, die das SOI legitimieren (weil sie es bauen und/oder einsetzen) sind bereit, die Verantwortung für die gewählten Wertprioritäten zu übernehmen, die sich an ihren eigenen Wertmaximen orientieren.

4. Die Organisation, die das SOI baut und/oder einsetzt, beachtet bei der Reihung der Werte die eigenen bereits vor- 
handenen Wertprinzipienlisten (etwa solche, die in CSRAnstrengungen formuliert wurden).

5. Die Organisation stellt sich durch ihr Geschäftsmodell und die Unternehmensstrategie (rund um das SOI) hinter die Wertprioritäten.

6. Umweltaspekte werden berïcksichtigt.

7. Bestehende Ethikstandards werden berücksichtigt (wie etwa die der eigenen Branche oder Menschenrechte).

Dem philosophisch geschulten Nutzer erschließt sich beim Lesen dieser sieben Kriterien der Wertereihung sofort, dass das zweite und dritte Kriterium der Kantschen Pflichtethik entliehen sind [15]. Wenn etwa die Automatisierung eines Prozesses einzig und allein zur Steigerung des Unternehmensprofits anstrebt wird (also des Geldwerts) und dadurch deutlich wird, dass freigesetzte Arbeiter lediglich als Mittel zum Produktionszweck benutzt werden, dann muss der Wert des Profits - so wichtig er fürs Unternehmen sein mag - aus ethischer Sicht hinter andere Werte gereiht werden. Zielt die Automatisierung jedoch klar darauf ab, Arbeitern die Arbeit zu erleichtern, dann werden sie nicht ausschließlich als Mittel zum Zweck der Einsparung angesehen und Profit kann höher gereiht werden.

Die Liste der 7 Kriterien berücksichtigt ebenso die von Unternehmen bereits erarbeiteten Werteprinzipienlisten. Es ist kritisiert worden, dass diese oft von CSR-Abteilungen erarbeiteten Listen wenig Eingang in gelebte Unternehmensprozesse finden. Durch VbE werden sie jedoch aufgerufen und können beeinflussen, welche SOI-Werte priorisiert werden sollten.

Schließlich sollte sichergestellt werden, dass Werteprioritäten mit externen Werterwartungen konform gehen. Externe Werteerwartungen sind etwa gesetzlich verankerte Werte oder internationale Abkommen zu ethischem Verhalten, wie die Menschenrechtskonvention der Vereinten Nationen. Sollte also ein Stakeholderteam tatsächlich den Wert des Trostes oder der Hilfe (im Modehausbeispiel) vor den der Privatsphäre gereiht haben, dann stellt der Abgleich mit der juristischen und politischen Außenwelt an dieser Stelle sicher, dass Privatsphäre doch vor dem Trost oder der Hilfe gereiht wird.

Am Ende der Werterhebung und der Priorisierungarbeit haben das Unternehmen und alle involvierten Parteien einen sehr genauen Einblick in die guten und schlechten Potenziale ihres geplanten SOI. Sollte sich dabei herausstellen, dass die Wertverletzungen überwiegen oder die positiven Werte letztlich wenig vielversprechend sind, im Vergleich zum Schaden, der entstehen kann, sollte sich das Projektteam und die Unternehmensleitung gegen eine Investition entscheiden. VbE fordert auf, den Verzicht auf eine Technologie aus ethischen Gründen aktiv in Erwägung zu ziehen. Fortschritt entsteht nicht dadurch, dass man Technologien in die Welt setzt, die mehr Schaden anrichten können, als sie Wertschöpfung für eine Gesellschaft versprechen (zum Verständnis des erweiterten Wertschöpfungsbegriffs siehe S. 27 in [23]).

\section{Übersetzung der Werte in Systemanforderungen}

Entscheidet sich ein Unternehmen für die Investition in ein SOI unter Berücksichtigung der öffentlich zu machenden Werteprioritäten, stellt sich als nächstes die Frage, wie die Wertstrategie systematisch im Systemdesign Eingang finden kann. Dazu wird zunächst für die priorisierten Wertcluster jedes Tupel „Kernwert/Wertqualität“ gesondert betrachtet und in ein sogenanntes „Ethical Value Requirement“ übersetzt (EVR); zu Deutsch ,ethische Wertanforderung“. EVRs, die streng genommen „Ethical Value Quality Requirements" heissen müssten, leiten sich direkt aus den Wertqualitäten ab. Es sind noch keine technischen Systemanforderungen, sondern genauer spezifizierte Aspekte eines technisch-organisatorischen Systems, die gegeben sein müssten, um eine Wertqualität in Erfüllung zu bringen; Anforderungen, könnte man sagen, die als Ziellinie für das ethisch fundierte Systemdesign dienen. Beispielsweise wären 5 EVRs für die ,informierte Zustimmung * (die in Abb 1a als Wertqualität von Privacy dargestellt ist): die verständliche und ehrliche, vollständige, einfach zugängliche Information zur tatsächlich stattfindenden Datenverarbeitung und die einfach handhabbare Nutzeroption, diese Datenverarbeitung jenseits des unbedingt Nötigen zu untersagen. Ein SOI, wie ein Sprachassistent, ist nur als ethisch anzusehen, wenn er diesen EVRs gerecht wird.

EVRs sind die Ausgangsbasis für die Ableitung von Systemanforderungen, die diese umsetzen sollen. Die oben genannten Beispiele zeigen jedoch, dass EVRs nicht nur von technischer, sondern auch von rein organisatorischer Natur sein können. Die Verständlichkeit, Ehrlichkeit und Vollständigkeit, mit der eine Sprachassistentenfirma ihre Datenverarbeitungspraktiken beschreiben sollte, um sich als ethisch betrachten zu dürfen, hat zunächst keinen technischen Charakter. Stattdessen ist die Unternehmensleitung dazu aufgefordert, zum Beispiel die Teilnahme an sogenannten „Personal Data Markets“ den NutzerInnen offen und einfach darzulegen. Eine andere Wertqualität befasst sich mit der Entscheidung, private Daten weiterzuverkaufen oder nicht. Ein EVR könnte sein, darauf zu verzichten. So ein Verzicht hat nichts mit technischem „Engineering“ zu tun, sondern ist „Organisational Engineering“ (siehe Weg 1 in Abb. 2).

Im Gegensatz dazu steht ein techniknahes EVR, das dazu auffordert, die Sicherheit des Sprachassistentennutzers zu gewährleisten. Hier fordert das Tupel „Kernwert Privatsphäre/Wertqualität Sicherheit“" das technische Engineering 
Abb. 2 Grober Ablauf der VbEMethode

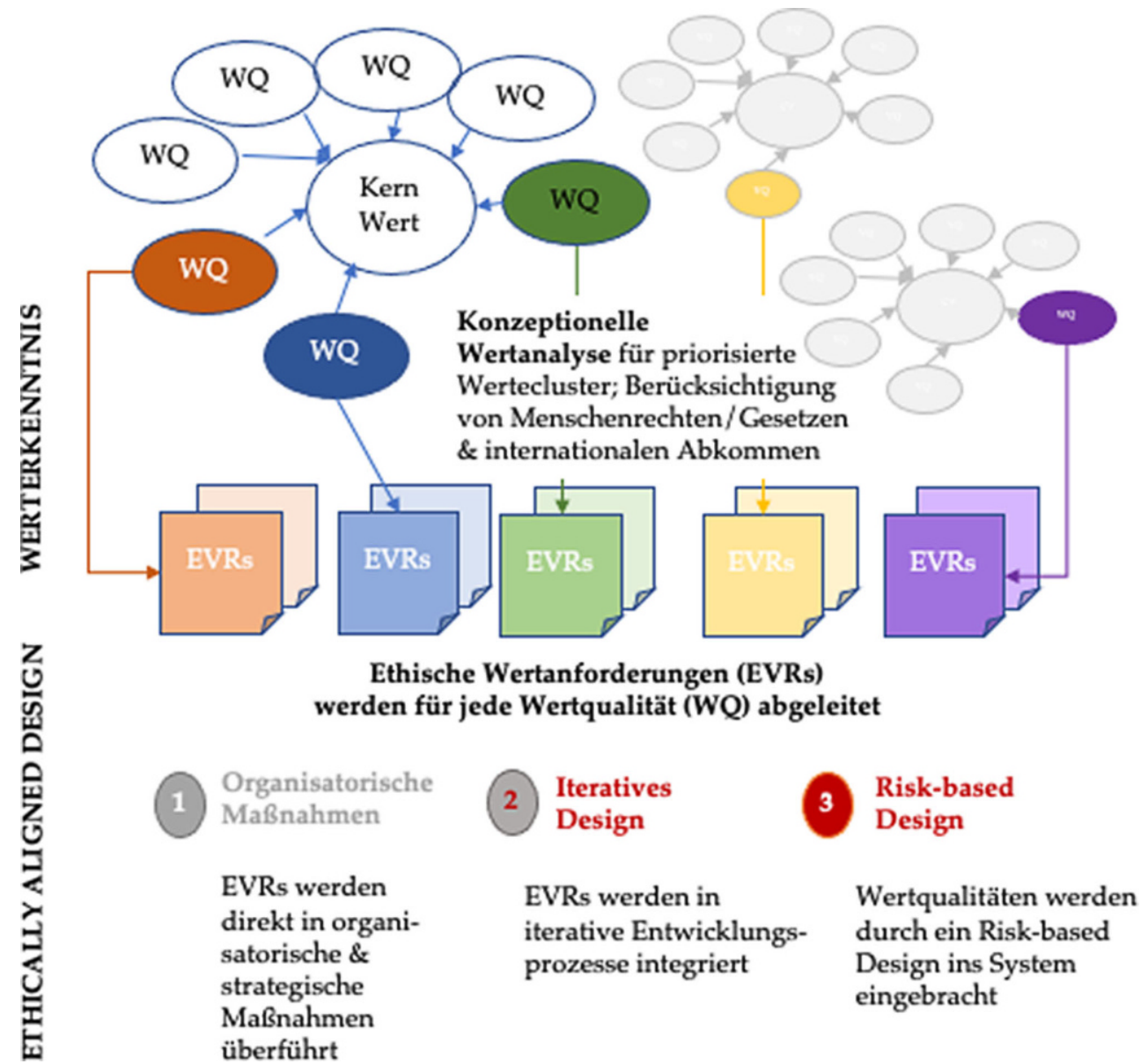

dazu auf, sich mit dem Wert so auseinanderzusetzen, dass die Kommunikation am Frontend und die Datenverarbeitung am Backend sicher gestaltet sind. Und da ein Mangel an Sicherheit dem Unternehmen und dem Nutzer einen erheblichen Schaden zufügen könnte, sollten die technischen Abteilungen ein hohes Eigeninteresse daran haben, beim Systemdesign keine sicherheitskritischen Anforderungen zu übersehen. Vor diesem Hintergrund rät das VbE einen rigorosen Risk-based-Design-Ansatz zu verfolgen. Rigoroses Risk-based-Design ist im Verständnis von VbE ein Verfahren zur Identifikation von Systemanforderungen, was sich insbesondere in den Bereichen der sicheren Technikgestaltung schon lange als Standard durchgesetzt hat $[5,18]$. Im VbE wird die Methodik dieses Ansatzes auf alle diejenigen Werte angewendet, die von besonders hoher Schutzbedeutung für Unternehmen und Stakeholder sind. Man stellt das Tupel „Kernwert/Wertqualität“ ins Zentrum der Schutzanalyse, indem man dieses als das Schutzziel definiert. Daran anschließend wird durchdacht, durch welches SOI-Design bzw. durch welche Schwachstellen eine Bedrohung des Werttupels entstehen könnte (die sogenannten „threats“ oder Angriffsmöglichkeiten auf das Wertziel, was es zu schützen gilt). Und schließlich überlegt man sich für je- de dieser Schwachstellen entsprechende Systemkontrollen. Letztere können dabei technischer oder organisatorischer Natur sein. Die technischen Systemkontrollen zum Schutz der Werte sind Systemanforderungen, die in den allgemeinen Pool der funktionalen Anforderungen an das System mit aufgenommen werden. So entsteht eine ethisch-funktional integrierte Product Roadmap (siehe Weg 3 in Abb. 2).

Wie aber geht man mit solchen Wertqualitäten um, deren EVRs keiner organisatorischen Natur sind und die in ihrer Beschaffenheit auch nicht so wichtig sind, dass man sie dem relativ aufwendigen Risk-based-Design unterziehen müsste? Ein Beispiel dafür ist der Sprachassistent, der dem Nutzer Mitgefühl vermitteln soll, um Trost zu spenden. Oder zu Beginn dieses Artikels wurde am Modehausbeispiel der Kernwert der Hilfe diskutiert, dessen Wertqualität u. a. darin besteht, den schnellen Zugang zu Verkäufern zu gewährleisten. So ein schneller Zugang muss im SOI (der Trackingapp) systemtechnisch abgebildet werden, ist aber in seinem Charakter so geartet, dass die aufwendigen Analysen des Risk-based-Design nicht nötig sind. Das käme einem Schießen von methodischen Kanonen auf Spatzen gleich. Vor diesem Hintergrund bietet das VbE im Gegensatz zu den Designprozessen des IEEE 7000 noch einen dritten 
Tab. 1 Die 10 Handlungsprinzipien des Value-based Engineering

Die 10 Handlungsprinzipien des Value-based Engineering

\begin{tabular}{ll}
\hline$(1)$ & Übernahme von Verantwortung für die erweiterte Systemlandschaft \\
$(2)$ & Ehrliche Integration von kritischen direkten und indirekten Stakeholdern \\
$(3)$ & Kontextsensitive, weise und kontinuierliche Beobachtung und Antizipation der Systementfaltung \\
$(4)$ & Nutzung ethischer und moralischer Prinzipien zum Erkennen dessen, was von Wert ist \\
$(5)$ & Konzeptionelle Analyse von dem, was von Wert ist (Werte in der Tiefe verstehen) \\
$(6)$ & Einbindung der Unternehmensführung in die Priorisierung von Werten \\
$(7)$ & Reflektion bestehender Gesetze und Abkommen zur Verfeinerung der Wertschwerpunkte \\
$(8)$ & Bereitschaft, auf das System zu verzichten, wenn es ethische Bedenken auslöst \\
$(9)$ & Bereitschaft, die eigene Wertestrategie offen zu legen und transparent zu leben \\
$(10)$ & Integration von Wertzielen in die Product Roadmap durch wertgeleitetes Systemdesign
\end{tabular}

Weg an, um EVRs im technischen System zu verankern. Dazu bedient man sich nichts anderem als den altbekannten, klassisch-iterativen Systementwicklungsverfahren. Das Design Thinking würde etwa vorsehen, dass ein ,first viable product" [4] - also ein noch recht unspezifischer Prototyp den Stakeholderrepräsentanten gezeigt wird und diese dann überlegen, wie sie im Interface den ,Zugang“ zu Personal am besten umsetzen könnten. Entscheidend ist hier, dass die interative Entwicklung des Prototyps durch Kernwerte und ihre Wertqualitäten ethisch angeleitet wird. Das Projektteam sucht also nicht ,Tabula rasa“ nach Nutzerbedürfnissen, sondern knüpft an die moralphilosophisch abgeleiteten Vorarbeiten an.

Abb. 2 illustriert den Fluss des VbE von der Herleitung der Wertprinzipien bis hin zu den praktischen Systemanforderungen. Der Kasten fasst die wesentlichen Prinzipien des VbE zusammen, die in diesem Artikel erläutert wurden und die in noch ausführlicherer Form auf dem laufend aktualisierten VbE-Informationsportal nachgelesen werden können. ${ }^{1}$

\section{Schlussfolgerungen und Herausforderungen des Value-based Engineering}

Die Beschreibung des VbE macht deutlich, dass es kein isolierter Unternehmensprozess ist, den man in eine Abteilung wie das Compliance-Management, Risk-Management oder die CSR-Abteilung auslagern kann. Ethik lässt sich nicht delegieren oder in eine Nischenfunktion auslagern. Damit „Gutes“ gelingt, muss Ethik als durchgängiges Prinzip in alle relevanten Innovationsprozesse eingewebt werden. VbE sieht daher vor, in frühen Phasen der Produktentwicklung die Wertethik und die Moralphilosophie als Hilfsmittel mit heranzuziehen, um die Werthaltigkeit der Unternehmensstrategie und der Produktgestaltung insgesamt zu beeinflussen. Das heißt letztlich, die „Value Proposition“ im Zen-

\footnotetext{
${ }^{1}$ https://www.wu.ac.at/value-based-engineering.
}

trum des Businessplans zu beeinflussen. Führungskräfte, Produktmanager und technische Abteilungen müssen dafür eng zusammenarbeiten, was heute nicht unbedingt immer selbstverständlich ist. Ebenso muss das Unternehmen, was aus Sicht des Kunden für das SOI verantwortlich ist, alle relevanten Servicepartner in das VbE einbinden, damit die Durchgängigkeit der Werteprinzipien gewährleistet ist. Das Ökosystem rund um das SOI (das sogenannte System of System) muss mit an Bord sein, um keine unerwarteten und unkontrollierbaren Werteuntergrabungen $\mathrm{zu}$ riskieren. Das bedeutet jedoch, dass SOI-Betreiber möglicherweise auf einige billige und liebgewonnene Supply-Chain-Partner verzichten müssen; keine einfache Entscheidung. Die Product Roadmap wird dann ebenso von Werteprioritäten beeinflusst, wie von rein funktionalen Systemanforderungen, was wiederum eine Investition in ein immateriell „,Gutes" bedeutet, die nicht jeder Kapitalgeber heute gerne sieht. Hinzu kommt, dass das ganze Engagement Zeit kostet, nicht nur um die hier beschriebene ethische Wertstrategie auszuarbeiten, sondern um diese auch systemtechnisch umzusetzen. Kurz: VbE verlangt ein fundamentales Umdenken in der Art und Weise, wie wir heute an die Systementwicklung herangehen. Es braucht mehr Zeit, mehr Geld, mehr Kooperation und Fürsorge für Stakeholder. Dass all das nicht einfach ist - egal ob durch IEEE standardisiert oder nicht - ist der Autorin dieses Artikels völlig klar. Es ist aber auch klar, dass eine menschengerechtere, soziale, qualitativ hochwertige und sozial/menschlich/gesellschaftlich gute Technik ohne ein radikales Umdenken unserer Innovationsprozesse nicht möglich sein wird. Wer sich für eine „bessere“ Welt einsetzt - und das ist letztlich das Grundanliegen der IT-Ethik-Bewegung - darf nicht glauben, dass er einfach so weitermachen kann wie bisher.

Fraglich ist natürlich, ob die Gesellschaft, die Unternehmen, die Verwaltung und die Politik heute schon bereit sind für dieses Umdenken. In der oben beschriebenen Telemedizin-Case-Study war der CEO nicht begeistert, all den negativen Wertpotenzialen seiner Plattform ins Gesicht zu sehen; geschweige denn Geld dafür in die Hand zu nehmen, die- 
se zu vermeiden. Kritik konstruktiv anzunehmen und Geld in Gutes zu investieren, wenn man sparsam sein muss, ist eine beträchtliche Ambition in der heutigen Zeit. Damit es überhaupt fruchten kann, muss VbE bzw. IEEE 7000 daher in so frühen Phasen der Systementwicklung ansetzen, wie möglich; am besten zu einem Zeitpunkt, wo die Wertstrategie und damit auch das Geschäftsmodell noch nicht festgefahren sind. Sollte es in einer Brownfield-Situation zum Einsatz kommen, muss unternehmensseitig eine hohe Bereitschaft zum Umdenken vorhanden sein.

Schließlich kommt zu der Reife, Bereitschaft und Fähigkeit, Feedback anzunehmen, und zu dem Mut, es sich von außenstehenden kritischen Stakeholdern einzuholen, noch eine weitere Herausforderung: Ethischer Erfolg lässt sich nicht genau messen. Dies ist nicht nur der Fall, weil abgewendete Angriffe ausbleiben und das Erfassen von Nichtgeschehenem „Bösen“ nicht möglich ist, sondern auch weil das Gute an sich nicht verlässlich messbar ist. Wie sollte man etwa messen, dass eine Plattform Wissen fördert? Man kann es nur vermuten. Selbst bei einer nicht wegzudenkenden Plattform wie Wikipedia diskutieren kritische Stimmen, ob sie immer Wissen bereitstellt und keinen Unsinn [24]. Aber kann man das messen und beweisen? Kernwerte sind sehr mannigfaltig und nur durch ihre Wertqualitäten im Kontext erfassbar, denn sie haben letztlich (wie Nikolai Hartmann schon feststellte [11]) die Natur der von Platon skizzierten ewigen Ideen. Sprich: Wenn wir dem gegenwärtigen Lauf der Geschichte weiter folgen, indem nur dem Messbaren eine Wirklichkeit zugesprochen wird, dann werden es wertebasierte Methoden in der Unternehmenspraxis schwer haben. VbE läutet also auch einen kulturellen Wandel für das Management ein. Es geht nicht mehr um Peter Druckers Sicht, dass nur das verwaltbar ist, was messbar ist, sondern es geht um die Einsicht, dass nur das erstrebenswert ist, was eine geteilte Werte- und damit Vertrauensbasis hat, auch wenn diese nicht messbar ist.

Danksagung Ich möchte an dieser Stelle den aktiven Teilnehmern der IEEE P7000 Working Group danken, deren Gedanken und Kritiken in diesem Artikel Widerhall finden, auch wenn nicht alles hier Dargestellte der Sicht von IEEE und der P7000 WG entspricht. Ebenso danke ich meinen Doktoranden Till Winkler, Esther Görnemann und Katrin Bednar für ihre Unterstützung der Case-Study-Analysen zu VbE sowie Feedback zu früheren Versionen des Artikels.

Funding Open access funding provided by Vienna University of Economics and Business (WU).

Open Access Dieser Artikel wird unter der Creative Commons Namensnennung 4.0 International Lizenz veröffentlicht, welche die Nutzung, Vervielfältigung, Bearbeitung, Verbreitung und Wiedergabe in jeglichem Medium und Format erlaubt, sofern Sie den/die ursprünglichen Autor(en) und die Quelle ordnungsgemäß nennen, einen Link zur Creative Commons Lizenz beifügen und angeben, ob Änderungen vorgenommen wurden.
Die in diesem Artikel enthaltenen Bilder und sonstiges Drittmaterial unterliegen ebenfalls der genannten Creative Commons Lizenz, sofern sich aus der Abbildungslegende nichts anderes ergibt. Sofern das betreffende Material nicht unter der genannten Creative Commons Lizenz steht und die betreffende Handlung nicht nach gesetzlichen Vorschriften erlaubt ist, ist für die oben aufgeführten Weiterverwendungen des Materials die Einwilligung des jeweiligen Rechteinhabers einzuholen.

Weitere Details zur Lizenz entnehmen Sie bitte der Lizenzinformation auf http://creativecommons.org/licenses/by/4.0/deed.de.

\section{Literatur}

1. Amazon Technologies (2018) USPTO

2. Aristoteles (1969) Nikomachische Ethik. Reclam Verlag, Stuttgart (F. Dirlmeier, Trans.)

3. Bednar K, Spiekermann S (2020) On the power of ethics: how value-based thinking fosters creative and sustainable IT product innovation (article in submission) Working paper. https://epub.wu.ac.at/ 7841/

4. Brown T (2008) Design thinking. Harv Bus Rev 86(6):84-92

5. BSI, Oetzel M, Kelter H, Spiekermann S, Mull S (2011) BSI PIA Leitfaden

6. Regulation (EU) 2016/679 Of The European Parliament And Of The Council of 27 April 2016on the protection of natural persons with regard to the processing of personal data and on the free movement of such data, and repealing Directive 95/46/EC (General Data Protection Regulation), L 119/1 C.F.R. (2016).

7. Friedman B, Hendry DG (2019) Value sensitive design: shaping technology with moral imagination. MIT Press,

8. Görnemann E, Spiekermann S (2020) Moments of truth with conversational agents: an exploratory quest for the relevant experiences of Alexa users. 28th European Conference on Information Systems (ECIS), Marakesh, June 15-17, 2020

9. Habermas J (1984) Reason and the Rationalization of Society. The Theory of Communicative Action, Bd. 1. Heinemann, London

10. Hao K (2020) The UK exam debacle reminds us that algorithms can't fix broken systems. MIIT Technology Review. https://www. technologyreview.com/2020/08/20/1007502/uk-exam-algorithmcant-fix-broken-system/. Zugegriffen: 20. Aug. 2020

11. Hartmann N (1935) Ethik, 4. Aufl. Walter de Gruyter, Berlin (1962)

12. IEEE (2021) IEEE 7000-Model Process for Addressing Ethical Concerns During System Design. IEEE Computer Society, Piscataway.

13. Jobin A, Ienca M, Vayena E (2019) The global landscape for AI ethics guidelines. Nature-Machine Intell 1:389-399

14. Kant I (1785) Groundwork for the metaphysics of morals. In: Gregor MJ, Wood AW (Hrsg) Practicle philosophy. Cambridge University Press, New York (1999, M. J. Gregor, Trans.)

15. Kant I (1786) Immanuel Kant's Werke. Suhrkamp,

16. Mill JS (1863) Utilitarianism. In: Ryan A (Hrsg) Utilitarianism and other essays. Penguin Books, London (1987)

17. Mingers J, Walsham G (2010) Toward ethical information systems: the contribution of discourse ethics. MISQ 34(4):833-854

18. National Institute for Standards and Technology (NIST) (2010) Risk Management Guide for IT Systems. http://csrc.nist.gov/ publications/nistpubs/800-30/sp800-30.pdf. Zugegriffen: 10. Juli 2021

19. Nonaka I, Kodama M, Hirose A, Kohlbacher F (2014) Dynamic fractal organizations for promoting knowledge-based transformation-A new paradigm for organizational theory. Eur Manag $\mathrm{J}$ 32:137-146

20. Publica P (2016) Machine Bias-There's software used across the country to predict future criminals. And it's biased against blacks 
21. Scheler M (1921) Der Formalismus in der Ethik und die Materiale Wertethik - Neuer Versuch der Grundlegung eines ethischen Personalismus, 2. Aufl. Verlag Max Niemeyer, Halle an der der Saale (2007, unveränderte Auflage)

22. Spiekermann S (2016) Ethical IT innovation-A value-based system design approach. CRC Press, Taylor \& Francis, New York, London and Boca Raton
23. Spiekermann S (2019) Digitale Ethik - Ein Wertesystem für das 21. Jahrhundert. Droemer, Munich

24. Wikipedia Community (2020) Criticism of Wikipedia. https://en. wikipedia.org/wiki/Criticism_of_Wikipedia\#cite_note-EdwinBlack1. Zugegriffen: 10. Juli 2021 